PUTP-94-20

\title{
GLUONIC AND LEPTONIC DECAYS OF HEAVY QUARKONIA AND THE DETERMINATION OF $\alpha_{s}\left(m_{c}\right)$ AND $\alpha_{s}\left(m_{b}\right)$
}

\author{
Kuang-Ta CHAO ${ }^{1,2}$ Han-Wen HUANG ${ }^{2}$ Yu-Quan LIU² \\ 1.CCAST (World Laboratory), Beijing 100080, P.R.China \\ 2.Department of Physics, Peking University, Beijing 100871, P.R.China
}

\begin{abstract}
QCD running coupling constant $\alpha_{s}\left(m_{c}\right)$ and $\alpha_{s}\left(m_{b}\right)$ are determined from heavy quarkonia $c \bar{c}$ and $b \bar{b}$ decays. The decay rates of $V \rightarrow 3 g$ and $V \rightarrow e^{+} e^{-}$ for $V=J / \psi$ and $\Upsilon$ are estimated by taking into account both relativistic and QCD radiative corrections. The decay amplitudes are derived in the BetheSalpeter formalism, and the decay rates are estimated by using the meson wavefunctions which are obtained with a QCD-inspired inter-quark potential. For the $V \rightarrow 3 g$ decay we find the relativistic correction to be very large and to severely suppress the decay rate. Using the experimental values of ratio $R_{g} \equiv \frac{\Gamma(V \longrightarrow 3 g)}{\Gamma\left(V \longrightarrow e^{+} e^{-}\right)} \approx 10,32$ for $V=J / \psi, \Upsilon$ respectively, and the calculated widths, we find $\alpha_{s}\left(m_{c}\right)=0.29 \pm 0.02$ and $\alpha_{s}\left(m_{b}\right)=0.20 \pm 0.02$. These values for the QCD running coupling constant are substantially enhanced, as compared with the ones obtained without relativistic corrections, and are consistent with the QCD scale parameter $\Lambda_{\overline{M S}}^{(4)} \approx 200 \mathrm{MeV}$. We also find that these results are mainly due to kinematic corrections and not sensitive to the dynamical models.
\end{abstract}




\section{INTRODUCTION}

Determining the QCD running coupling constant $\alpha_{s}$ at different energy scales is very important in the verification of the fundamental theory of strong interaction. Among others the heavy quarkonia decays may provide very useful information for $\alpha_{s}$ at the heavy quark mass scale. Decay rates of heavy quarkonia in the nonrelativistic limit with QCD radiative corrections have been studied (see, e.g., refs.[1,2,3]). However, the decay rates of many processes are subject to substantial relativictic corrections. In particular, the rate of $J / \psi \rightarrow 3 g$ and accordingly the determination of $\alpha_{s}\left(m_{c}\right)$ depend rather crucially on the relativistic corrections. Presently the ratios of gluonic to leptonic widths of $J / \psi$ and $\Upsilon$ have been precisely measured in experiment. However, if using the non-relativistic expressions for decay widths which are proportional to $\left|R_{S}(0)\right|^{2}$, where $\left|R_{S}(0)\right|$ is the radial wavefunction at the origin, and comparing them with the corresponding experimental values, one would get ${ }^{[2,3]}$ $\alpha_{s}\left(m_{c}\right)=0.19$ and $\alpha_{s}\left(m_{b}\right)=0.17$. They are substantially smaller than the expected values determined from other experimental results or the QCD scale parameter (for a review, see ref.[4]). Theoretically, the difficulty is mainly due to the large relativistic effects on the decay widths. It is well known that there are at least two important sources of relativistic effects for these processes, one is from the kinematical corrections to decay amplitudes, the other is from the bound state wavefunction corrections which are concerned with the dynamical effect of quark-antiquark interactions. In ref.[5], the first order relativistic corrections were considered based on a phenomenological model. But only the relativistic correction originated from kinematics was discussed and no explicit methods with dynamical considerations were given to calculate the decay widths. As a result of lack of reliable estimates for relativistic corrections to the decay widths in the determination of $\alpha_{s}\left(m_{c}\right)$ and $\alpha_{s}\left(m_{b}\right)$, either an arbitrary choice for the $v^{2} / c^{2}$ term is made to get these coupling constants enhanced $^{[3]}$, or the $v^{2} / c^{2}$ term is arbitrarily neglected but a large effective mass for the gluons is introduced ${ }^{[6]}$. Apparently, a better estimate rather than arbitrary guesses for the relativistic corrections is indeed needed, though this is certainly a very difficult task without a deep understanding of quark confinement.

In this paper, as an attempt to tackle this problem, we will use the Bethe-Salpeter (BS) formalism to derive the decay amplitudes and to calculate the decay widths of $V \rightarrow e^{+} e^{-}$and $V \rightarrow 3 g$, where $V$ is a vector heavy quarkonium state. In this approach, the meson is considered as a bound state consist of a pair of constitute quark and antiquark, and described by the BS wavefunction which satisfies the BS equation. A phenomenological QCD-inspired interquark potential will be used to solve for the wavefunctions. These may allow us to give the expressions which take into account both relativistic and QCD radiative corrections to next-to-leading order. We may then estimate both kinematical and dynamical relativistic corrections to the decay widths, and determine the QCD coupling constant from the calculated ratios of gluonic to leptonic decay widths. The remainder of this paper is organized as follows. 
In Sec.2 we study the relativistic corrections to $V \rightarrow e^{+} e^{-}$and $V \rightarrow 3 g$ widths. In Sec.3 we discuss the bound state wavefunctions and determine the coupling constants $\alpha_{s}\left(m_{c}\right)$ and $\alpha_{s}\left(m_{b}\right)$ by using the experimental values of the gluonic and leptonic widths of $J / \psi$ and $\Upsilon$. A summary and discussion will be given in the last section.

\section{RELATIVISTIC CORRECTIONS TO $V \rightarrow e^{+} e^{-}$AND $V \rightarrow 3 g$}

(1). The $V \rightarrow e^{+} e^{-}$decay

We first consider the leptonic decay for $V=J / \psi$ and $\Upsilon$ (see also ref.[7]). This process proceeds via the $Q \bar{Q}$ annihilation. Define the Bethe-Salpeter wavefunction, in general, for a $Q_{1} \bar{Q}_{2}$ bound state $\mid P>$ with overall mass $M$ and momentum $P=$ $\left(\sqrt{\vec{P}^{2}+M^{2}}, \vec{P}\right)$

$$
\chi\left(x_{1}, x_{2}\right)=<0\left|T \psi_{1}\left(x_{1}\right) \bar{\psi}_{2}\left(x_{2}\right)\right| P>,
$$

where $T$ represents time-order product, and transform it into momentum space

$$
\chi_{P}(q)=e^{-i P \cdot X} \int d^{4} x e^{-i q \cdot x} \chi\left(x_{1}, x_{2}\right) .
$$

Here $q_{1}\left(m_{1}\right)$ is the quark momentum (mass), $q_{2}\left(m_{2}\right)$ the antiquark momentum (mass), and $q$ the relative momentum,

$$
\begin{array}{r}
X=\eta_{1} x_{1}+\eta_{2} x_{2}, \quad x=x_{1}-x_{2}, \\
P=q_{1}+q_{2}, \quad q=\eta_{2} q_{1}-\eta_{1} q_{2},
\end{array}
$$

where $\eta_{i}=\frac{m_{i}}{\left(m_{1}+m_{2}\right)}(i=1,2)$.

In this formalism the quarkonium annilination matrix elements can be written as

$$
\langle 0|\bar{Q} I Q| P\rangle=\int d^{4} q \operatorname{Tr}\left[I(q, P) \chi_{P}(q)\right],
$$

where $I(q, P)$ is the interaction vertex of the $Q \bar{Q}$ with other fields (e.g., the photons or gluons ) which, in general, may also depend on the variable $q^{0}$. If $I(q, P)$ is independent of $q^{0}$, Eq. (4) can be written as

$$
\langle 0|\bar{Q} I Q| P\rangle=\int d^{3} q \operatorname{Tr}\left[I(\vec{q}, P) \Phi_{P}(\vec{q})\right]
$$

where

$$
\Phi_{P}(\vec{q})=\int d q^{0} \chi_{P}(q)
$$

is the three dimensional BS wave function of the $Q \bar{Q}$ meson. In the BS formalism the wavefunctions in the meson rest frame, where $\vec{q}_{1}=-\vec{q}_{2}=\vec{q}, P=(M, 0)$, can be expressed as

$$
\begin{aligned}
& \Phi_{P}^{0^{-}}(\vec{q})=\Lambda_{+}^{1}(\vec{q}) \gamma^{0}\left(1+\gamma^{0}\right) \gamma_{5} \gamma^{0} \Lambda_{-}^{2}(-\vec{q}) \varphi(\vec{q}) \\
& \Phi_{P}^{1^{-}}(\vec{q})=\Lambda_{+}^{1}(\vec{q}) \gamma^{0}\left(1+\gamma^{0}\right) \notin \gamma^{0} \Lambda_{-}^{2}(-\vec{q}) f(\vec{q})
\end{aligned}
$$


where $\Phi_{P}^{0^{-}}(\vec{q})$, and $\Phi_{P}^{1^{-}}(\vec{q})$ represent the three dimensional wave functions of $0^{-}$and $1^{-}$mesons respectively, $\phi=e_{\mu} \gamma^{\mu}, e_{\mu}$ is the polarization vector of $1^{-}$meson, $\varphi$ and $f$ are scalar functions which can be obtained by solving the BS equation for $0^{-}$and $1^{-}$ mesons, and $\Lambda_{+}\left(\Lambda_{-}\right)$are the positive (negative) energy projector operators

$$
\begin{aligned}
& \Lambda_{+}^{1}(\vec{q})=\Lambda_{+}\left(\vec{q}_{1}\right)=\frac{1}{2 E}\left(E+\gamma^{0} \vec{\gamma} \cdot \vec{q}_{1}+m \gamma^{0}\right), \\
& \Lambda_{-}^{2}(-\vec{q})=\Lambda_{-}\left(\vec{q}_{2}\right)=\frac{1}{2 E}\left(E-\gamma^{0} \vec{\gamma} \cdot \vec{q}_{2}-m \gamma^{0}\right), \\
& E=\sqrt{\vec{q}^{2}+m^{2}} .
\end{aligned}
$$

For process $V \rightarrow e^{+} e^{-}$with the electron (positron) momentum $p_{1}\left(p_{2}\right)$ and helicity $r_{1}\left(r_{2}\right)$,

$$
I(\vec{q}, P)=-i e \gamma_{\mu},
$$

and the amplitude can be written as

$$
T=e^{2} e_{Q}\left\langle 0\left|\bar{Q} \gamma_{\mu} Q\right| V\right\rangle \bar{u}_{r_{1}}\left(p_{1}\right) \gamma^{\mu} v_{r_{2}}\left(p_{2}\right) \frac{1}{M^{2}}
$$

where $M$ is the meson mass and $e_{Q}$ is the electric charge of the quark $Q(Q=c, b)$. Define the decay constant $f_{V}$ by

$$
f_{V} M e_{\mu} \equiv\left\langle 0\left|\bar{Q} \gamma_{\mu} Q\right| V\right\rangle=\int d^{3} q \operatorname{Tr}\left[\gamma_{\mu} \Phi_{P}(\vec{q})\right]
$$

where $e_{\mu}$ is the polarization vector of $V$ meson. Then with (7) we can easily find

$$
f_{V}=\frac{2 \sqrt{3}}{M} \int d^{3} q\left(\frac{m+E}{E}-\frac{\vec{q}^{2}}{3 E^{2}}\right) f(\vec{q}),
$$

where $E=\sqrt{\vec{q}^{2}+m^{2}}$, and $\sqrt{3}$ is the color factor. Summing over the polarizations of the final state and averaging over that of the initial state, it is easy to get the decay width

$$
\Gamma\left(V \rightarrow e^{+} e^{-}\right)=\frac{4}{3} \pi \alpha^{2} e_{Q}^{2} f_{V}^{2} / M
$$

Including further the QCD radiative correction and assuming that the radiative correction and relativistic correction can be factorized, we then get the following expression for the decay width with both relativistic and QCD radiative corrections

$$
\Gamma\left(V \rightarrow e^{+} e^{-}\right)=\frac{4}{3} \pi \alpha^{2} e_{Q}^{2} f_{V}^{2} / M\left(1-\frac{16 \alpha_{s}\left(m_{Q}\right)}{3 \pi}\right) .
$$

Expanding $f_{V}$ in terms of $\vec{q}^{2} / m^{2}$, to the first order we get

$$
f_{V}=-\frac{4 \sqrt{3}}{M} \int d \vec{q} f(\vec{q})\left(1-\frac{5}{12} \frac{\vec{q}^{2}}{m^{2}}\right) .
$$


2. The $V \rightarrow 3 g$ decay.

We next study the hadronic decay for $V=J / \psi$ and $\Upsilon$. We consider a $J^{P C}=1^{--}$ $Q \bar{Q}$ bound state decaying into three gluons. The decay width is given by

$$
\Gamma=\frac{1}{2 M} \int d \phi Z,
$$

where the integration $\int d \phi$ is over the final-state phase space. $Z$ is defined by

$$
Z=\sum|T|^{2},
$$

where $\sum$ represents summing over the polarizations of the final state and averaging over that of the initial state. The decay matrix element $T$ is

$$
T=-i g_{s}^{3} \operatorname{Tr}\left(T_{a} T_{b} T_{c}\right) \int d^{4} q \operatorname{Tr} \chi_{P}(q) I\left(k_{1}, k_{2}, k_{3} ; q_{1}, q_{2}\right),
$$

where

$$
\begin{aligned}
I\left(k_{1}, k_{2}, k_{3} ; q_{1}, q_{2}\right)= & \frac{\phi_{3}\left(\not k 3-\not q_{2}+m\right) \phi_{2}\left(\phi_{1}-\not k_{1}+m\right) \phi_{1}}{\left(\left(k_{3}-q_{2}\right)^{2}-m^{2}\right)\left(\left(q_{1}-k_{1}\right)^{2}-m^{2}\right)} \\
& + \text { all permutations of } 1,2,3 .
\end{aligned}
$$

Here $k_{i}$ and $\epsilon_{i}(i=1,2,3)$ represent the momenta and polarizations of the three gluons; $T_{a}, T_{b}, T_{c}$ are the color $S U(3)$ matrices, and $a, b, c$ are the color indices of the three gluons; $q_{1}$ and $q_{2}$ are the momenta of quark and antiquark, and their time components satisfy $q_{1}^{0}+q_{2}^{0}=M$. As usual, we assume $e^{[1,5]}$

$$
q^{0}=q_{1}^{0}-q_{2}^{0}=0, \quad q_{1}^{0}=q_{2}^{0}=M / 2 .
$$

Thus $I\left(k_{1}, k_{2}, k_{3}, q_{1}, q_{2}\right)$ is independent of $q^{0}$, and $T$ becomes

$$
T=-i g_{s}^{3} \operatorname{Tr}\left(T_{a} T_{b} T_{c}\right) \int d^{3} q \operatorname{Tr}\left\{\Phi_{P}^{1^{-}}(\vec{q}) I\left(k_{1}, k_{2}, k_{3} ; \vec{q}\right)\right\} .
$$

Substituting (7) and (19) into (21) and (17), we get the expression for $Z$. In the extremely non-relativistic limit the dependence on $\vec{q}$ of $\Lambda_{+}(\vec{q}), \Lambda_{-}(\vec{q})$ and $I\left(k_{1}, k_{2}, k_{3} ; \vec{q}\right)$ in $Z$ is neglected and we obtain the usual non-relativistic formula for $V \rightarrow 3 g$. In order to go beyond the leading order result we expand the matrix element $T$ in terms of $\vec{q}^{2} / m^{2}$. After performing the trace of $\gamma$ matrix, for $m=M / 2$ we get to the first order of $v^{2} / c^{2}$

$$
\begin{aligned}
Z=\quad & \frac{160 g_{s}{ }^{6}}{81 M^{4}} \int d^{3} q d^{3} q^{\prime}\left\{A_{0}\left(x_{1}, x_{2}, x_{3}\right)\left(1+\frac{\vec{q}^{2}+\vec{q}^{2}}{6 m^{2}}\right)\right. \\
& \left.+A_{1}\left(x_{1}, x_{2}, x_{3}\right) \frac{\vec{q}^{2}+\vec{q}^{2}}{3 m^{2}}\right\} f(\vec{q}) f\left(\vec{q}^{\prime}\right),
\end{aligned}
$$


where $f(\vec{q})$ is the scalar wavefunction of vector meson which comes from (7), and where

$$
\begin{aligned}
A_{0}\left(x_{1}, x_{2}, x_{3}\right)= & \frac{16}{x_{1}^{2}}+\frac{16}{x_{2}^{2}}+\frac{32}{x_{1} x_{2}}-\frac{32}{x_{1}^{2} x_{2}}-\frac{32}{x_{1} x_{2}^{2}}+\frac{16}{x_{1}^{2} x_{2}^{2}} \\
& +(\text { two other permutations }), \\
A_{1}\left(x_{1}, x_{2}, x_{3}\right)=\quad & -\frac{16}{x_{1}^{2}}-\frac{16}{x_{2}^{2}}-\frac{41}{x_{1} x_{2}}+\frac{12}{x_{1}^{2} x_{2}}+\frac{12}{x_{1} x_{2}^{2}}-\frac{40}{x_{1}^{2} x_{2}^{2}} \\
& -\frac{8}{x_{1}^{3}}-\frac{8}{x_{2}^{3}}-\frac{8}{x_{1}^{3} x_{2}}-\frac{8}{x_{1} x_{2}^{3}}+\frac{48}{x_{1}^{3} x_{2}^{2}}+\frac{48}{x_{1}^{2} x_{2}^{3}}-\frac{32}{x_{1}^{3} x_{2}^{3}} \\
& +(\text { two other permutations }), \\
& \frac{2 \omega_{i}}{M}, \quad i=1,2,3 .
\end{aligned}
$$

where $\omega_{i}$ represents the gluonic energy. The final-state phase space is given by

$$
d \phi=(2 \pi)^{4} \delta^{4}\left(P-\sum_{i=1}^{3} k_{i}\right) \prod_{i=1}^{3} \frac{d^{3} k_{i}}{(2 \pi)^{3} 2 \omega_{i}} .
$$

After performing the integration, we find the decay width of $V \rightarrow 3 g$ with relativistic corrections to be

$$
\Gamma(V \rightarrow 3 g)=\frac{640\left(\pi^{2}-9\right) \alpha_{s}^{3}\left(m_{Q}\right)}{81 M^{3}} g_{V}^{2}
$$

where to the first order relativistic correction $g_{V}$ is given by

$$
\begin{aligned}
g_{V} & =\int d^{3} q\left[1-\left(\frac{\left(41 \pi^{2} / 3-48\right)}{32\left(\pi^{2}-9\right)}-1 / 6\right) \frac{\vec{q}^{2}}{m^{2}}\right] f(\vec{q}) \\
& =\int d^{3} q\left[1-2.96 \frac{\vec{q}^{2}}{m^{2}}\right] f(\vec{q}) .
\end{aligned}
$$

This integral will diverge when the wavefunction $f$ is determined by the inter-quark potential which is Coulombic at short distances where one gluon-exchange dominates. We could introduce a cut-off scale $\Lambda$ of order $m(v / c)$ to regularize the integral. However, examining the interaction vertex $I$ given in (19) we can easily see that $g_{V}$ should be a convergent quantity if all higher order corrections are taken into consideration, because in (19) $I$ becomes inversely proportional to $\vec{q}^{2}$ as $\vec{q}^{2} \rightarrow \infty$. In the absence of calculations for higher order corrections we will take the following expression for the regulation of $g_{V}$

$$
g_{V} \approx \int d^{3} q\left[1+2.96 \frac{\vec{q}^{2}}{m^{2}}\right]^{-1} f(\vec{q})
$$

which gives the same result as (26) to the first order relativistic correction. Similarly, for the leptonic decay the first order expression of decay constant $f_{V}$ in (15) may also be regularized in the same manner, which differs from the full expression (12) only by higher order terms, and the difference turned out to be small. 
In connection with (20), another scheme is to take the on-shell condition, which assumes (see, e.g., ref.[5]) the quark and antiquark to be on the mass shell

$$
q_{1}^{0}=q_{2}^{0}=M / 2=E=\sqrt{m^{2}+\vec{q}^{2}} .
$$

The advantage of this assumption is that the gauge invariance is maintained for the on-shell quarks but at the price of treating the quark and antiquark just as free particles in a bound state. An apparent problem in this scheme is that if the quark mass takes a fixed value (as in the case before) then $\vec{q}^{2}$ will be fixed but not weighted by the wavefunction as in the usual bound state description. In order to connect the decay process, which occurs at short distances, where quarks are approximately on shell, with the bound state wavefunction, which is mainly determined by the long distance confinement force, we have to make a compromise between the on-shell condition and the bound state description. We will expand the matrix element (21) and (17) in terms of $\frac{\vec{q}^{2}}{E^{2}}=\frac{\vec{q}^{2}}{(M / 2)^{2}}$, and allow $\vec{q}^{2}$ (so the quark mass accordingly) to vary in accordance with the bound state wavefunction $f(\vec{q})$ which is to be determined by the long distance dynamics, or phenomenologically by some dynamical models. With this treatment, we get another expression for $Z$

$$
\begin{aligned}
Z=\quad & \frac{160 g_{s}{ }^{6}}{81 M^{4}} \int d^{3} q d^{3} q^{\prime}\left\{A_{0}\left(x_{1}, x_{2}, x_{3}\right)\left(1-\frac{5 \vec{q}^{2}+5 \vec{q}^{2}}{6(M / 2)^{2}}\right)\right. \\
& \left.+A_{1}\left(x_{1}, x_{2}, x_{3}\right) \frac{\vec{q}^{2}+\vec{q}^{2}}{3(M / 2)^{2}}\right\} f(\vec{q}) f\left(\vec{q}^{\prime}\right),
\end{aligned}
$$

where

$$
\begin{aligned}
A_{0}\left(x_{1}, x_{2}, x_{3}\right)= & \frac{16}{x_{1}^{2}}+\frac{16}{x_{2}^{2}}+\frac{32}{x_{1} x_{2}}-\frac{32}{x_{1}^{2} x_{2}}-\frac{32}{x_{1} x_{2}^{2}}+\frac{16}{x_{1}^{2} x_{2}^{2}} \\
& +(\text { two other permutations }), \\
A_{1}\left(x_{1}, x_{2}, x_{3}\right)= & \frac{20}{x_{1}^{2}}+\frac{20}{x_{2}^{2}}+\frac{28}{x_{1} x_{2}}-\frac{56}{x_{1}^{3} x_{2}}-\frac{56}{x_{1} x_{2}^{3}}-\frac{88}{x_{1}^{2} x_{2}^{2}} \\
& +\frac{72}{x_{1}^{3} x_{2}^{2}}+\frac{72}{x_{1}^{2} x_{2}^{3}}+\frac{16}{x_{1}^{3}}+\frac{16}{x_{2}^{3}}-\frac{32}{x_{1}^{3} x_{2}^{3}} \\
& +(\text { two other permutations }) .
\end{aligned}
$$

Then to the first order relativistic correction, we get

$$
\begin{aligned}
g_{V} & =\int d^{3} q f(\vec{q})\left[1-\left(\frac{36-\frac{7}{3} \pi^{2}}{8\left(\pi^{2}-9\right)}+\frac{5}{6}\right) \frac{\vec{q}^{2}}{(M / 2)^{2}}\right] \\
& \approx \int d^{3} q f(\vec{q})\left[1+2.7 \frac{\vec{q}^{2}}{(M / 2)^{2}}\right]^{-1}
\end{aligned}
$$

where $g_{V}$ has been regulated in the same manner as (27). 
Including futher the QCD radiative correction and assuming again that the radiative and relativistic corrections can be factorized, we then get the following expression for the decay width with both relativistic and QCD radiative corrections

$$
\Gamma(V \rightarrow 3 g)=\frac{640\left(\pi^{2}-9\right) \alpha_{s}^{3}\left(m_{Q}\right)}{81 M^{3}}\left(1-C \frac{\alpha_{s}\left(m_{Q}\right)}{\pi}\right) g_{V}^{2},
$$

where $C=3.7(4.9)$ for $Q=c(b)^{[2,3]}$. In the extremely non-relativistic limit, the $\vec{q}^{2} / m^{2}$ term is zero, and the scalar wavefunction $f(q)$ satisfies the following relation

$$
\int d^{3} q f(\vec{q})=\sqrt{\frac{M}{4}} \psi(0),
$$

where $\psi(0)$ is the nonrelativistic wavefunction at the origin in coordinate space, we then get the well-known zero-order result ${ }^{[2,3,5]}$

$$
\Gamma(V \rightarrow 3 g)=\frac{160\left(\pi^{2}-9\right) \alpha_{s}^{3}\left(m_{Q}\right)}{81 M^{3}}|\psi(0)|^{2} .
$$

With the two expressions (26) and (31), obtained in the two different treatments concerning the on-shell condition, we find that they both give very close results. This may largely reduce the uncertainties in our calculations associated with the onshell or off-shell descriptions for the quarks which decay at short distances (therefore approximately on shell) and are bound together at large distances (therefore off shell).

Comparing (26) and (31) with (15), we see that the suppression due to relativistic correction for $V \rightarrow 3 g$ is much more severe than for $V \rightarrow e^{+} e^{-}$. This result then rules out the conjecture that the relativistic correction to $J / \psi \rightarrow 3 g$ may be neglibibly small ${ }^{[6]}$.

\section{DETEMINATION OF $\alpha_{s}\left(m_{Q}\right)$ AND BOUND STATE WAVE FUNC- TIONS}

To calculate the widths of leptonic and gluonic decays, we have to know the wavefunctions $f(\vec{q})$ for $c \bar{c}$ and $b \bar{b}$ states, which are determined mainly by the long distance inter-quark dynamics. In the absence of a deep understanding for quark confinement at present, we will follow a phenomenological approach by using QCD inspired inter-quark potentials, which are supported by both lattice QCD calculations and heavy quark phenomenology, as the interaction kernel in the BS equation. We begin with the bound state BS equation ${ }^{[8]}$ in momentum space

$$
\left(\not q_{1}-m_{1}\right) \chi_{P}(q)\left(\not_{2}+m_{2}\right)=\frac{i}{2 \pi} \int d^{4} k G(P, q-k) \chi_{P}(k),
$$

where $q_{1}$ and $q_{2}$ represent the momenta of quark and antiquark respectively, $G(P, q-$ $k)$ is the interaction kernel which dominates the inter-quark dynamics. In solving 
Eq.(35), in order to avoid the notorious problem due to the excitation of relative time variable we have to employ the "instantaneous approximation". Meanwhile, we will neglect negative energy projectors in the quark propagators. We then get the reduced Salpeter equation $^{[8]}$ for the three dimensional BS wavefunction $\Phi_{P}(\vec{q})$ defined in (6)

$$
\Phi_{P}(\vec{q})=\frac{1}{P^{0}-E_{1}-E_{2}} \Lambda_{+}^{1} \gamma^{0} \int d^{3} k G(P, \vec{q}-\vec{k}) \Phi_{P}(\vec{k}) \gamma^{0} \Lambda_{-}^{2},
$$

where $G(P, \vec{q}-\vec{k})$ represents the instantaneous potential. We employ the following interquark potrntials including a long-ranged confinement potential (Lorentz scalar) and a short-ranged one-gluon exchange potential (Lorentz vector) ${ }^{[7]}$

$$
\begin{aligned}
& V(r)=V_{S}(r)+\gamma_{\mu} \otimes \gamma^{\mu} V_{V}(r), \\
& V_{S}(r)=\lambda r \frac{\left(1-e^{-\alpha r}\right)}{\alpha r}, \\
& V_{V}(r)=-\frac{4}{3} \frac{\alpha_{s}(r)}{r} e^{-\alpha r},
\end{aligned}
$$

where the introduction of the factor $e^{-\alpha r}$ is to regulate the infrared divergence and also to incorporate the color screening effects of the dynamical light quark pairs on the $Q \bar{Q}$ linear confinement potential ${ }^{[9]}$. In momentum space the potentials become ${ }^{[7]}$

$$
\begin{aligned}
& G(\vec{p})=G_{S}(\vec{p})+\gamma_{\mu} \otimes \gamma^{\mu} G_{V}(\vec{p}), \\
& G_{S}(\vec{p})=-\frac{\lambda}{\alpha} \delta^{3}(\vec{p})+\frac{\lambda}{\pi^{2}} \frac{1}{\left(\vec{p}^{2}+\alpha^{2}\right)^{2}} \\
& G_{V}(\vec{p})=-\frac{2}{3 \pi^{2}} \frac{\alpha_{s}(\vec{p})}{\vec{p}^{2}+\alpha^{2}}
\end{aligned}
$$

where $\alpha_{s}(\vec{p})$ is the quark-gluon running coupling constant and is assumed to become a constant of $O(1)$ as $\vec{p}^{2} \rightarrow 0$

$$
\alpha_{s}(\vec{p})=\frac{12 \pi}{27} \frac{1}{\ln \left(a+\vec{p}^{2} / \Lambda_{Q C D}^{2}\right)} .
$$

The constants $\lambda, \alpha, a$ and $\Lambda_{Q C D}$ are the parameters that characterize the potential. Substituting (7) and (38) into Eq.(36), one derives the equation for the $1^{-}$meson wavefunction $f(\vec{q})$ in the meson rest frame ${ }^{[7]}$

$$
\begin{aligned}
M f_{1}(\vec{q})= & \left(E_{1}+E_{2}\right) f_{1}(\vec{q}) \\
& -\frac{1}{4 E_{1} E_{2}} \int d^{3} k\left(G_{S}(\vec{q}-\vec{k})-2 G_{V}(\vec{q}-\vec{k})\right)\left(E_{1} m_{2}+E_{2} m_{1}\right) f_{1}(\vec{k}) \\
& -\frac{E_{1}+E_{2}}{4 E_{1} E_{2}} \int d^{3} k G_{S}(\vec{q}-\vec{k}) \frac{E_{1} m_{2}+E_{2} m_{1}}{m_{1}+m_{2}} f_{1}(\vec{k})
\end{aligned}
$$




$$
\begin{aligned}
& +\frac{E_{1} E_{2}-m_{1} m_{2}+\vec{q}^{2}}{4 E_{1} E_{2} \vec{q}^{2}} \int d^{3} k\left(G_{S}(\vec{q}-\vec{k})+4 G_{V}(\vec{q}-\vec{k})\right)(\vec{q} \cdot \vec{k}) f_{1}(\vec{k}) \\
& -\frac{E_{1} m_{2}-E_{2} m_{1}}{4 E_{1} E_{2} \vec{q}^{2}} \int d^{3} k\left(G_{S}(\vec{q}-\vec{k})-2 G_{V}(\vec{q}-\vec{k})\right)(\vec{q} \cdot \vec{k}) \frac{E_{1}-E_{2}}{m_{1}+m_{2}} f_{1}(\vec{k}) \\
& -\frac{E_{1}+E_{2}-m_{1}-m_{2}}{2 E_{1} E_{2} \vec{q}^{2}} \int d^{3} k G_{S}(\vec{q}-\vec{k})(\vec{q} \cdot \vec{k})^{2} \frac{1}{E_{1}+E_{2}+m_{1}+m_{2}} f_{1}(\vec{k}) \\
& -\frac{m_{1}+m_{2}}{E_{1} E_{2} \vec{q}^{2}} \int d^{3} k G_{V}(\vec{q}-\vec{k})(\vec{q} \cdot \vec{k})^{2} \frac{1}{E_{1}+E_{2}+m_{1}+m_{2}} f_{1}(\vec{k}),
\end{aligned}
$$

where

$$
f_{1}(\vec{q})=-\frac{m_{1}+m_{2}+E_{1}+E_{2}}{4 E_{1} E_{2}} f(\vec{q}) .
$$

The normalization condition of the wavefunction $f(\vec{q})$ reads

$$
\int d^{3} q \frac{2 E_{1} E_{2}\left(m_{1}+E_{1}\right)\left(m_{2}+E_{2}\right)}{\left(E_{1}+E_{2}+m_{1}+m_{2}\right)^{2}} f_{1}^{2}(\vec{q})=\frac{M}{4 \pi^{3}}
$$

Eq.(40) looks very complicated since higher order terms are all involved. To the leading order in the nonrelativistic limit, Eq.(40) is just the ordinary nonrelativistic Schrodinger equation. To the first order of $v^{2} / c^{2}$, Eq.(40) becomes the well known Breit equation with both vector (one-gluon) exchange and scalar (confinement) exchange.

For the heavy quarkonium $c \bar{c}$ and $b \bar{b}$ systems, $m_{1}=m_{2}=m$, Eq.(40) can be greatly simplified. By solving Eq.(40) we can find the wave functions for the $1^{-}$ mesons (see, e.g., ref.[7]).

Substituting the obtained BS wave functions into (12) and (14), we then get

$$
\Gamma\left(J / \psi \longrightarrow e^{+} e^{-}\right)=5.6 k e V,
$$

where we have used

$$
\begin{aligned}
& m_{c}=1.5 \mathrm{GeV}, \quad \lambda=0.23 \mathrm{GeV}^{2}, \quad \Lambda_{Q C D}=0.18 \mathrm{GeV}, \\
& \alpha=0.06 \mathrm{GeV}, \quad a=e=2.7183 .
\end{aligned}
$$

With these parameters the $2 S-1 S$ spacing and $J / \psi-\eta_{c}$ splitting are required to fit the data. Our result is in agreement with the experimental value of $\Gamma(J / \psi \longrightarrow$ $\left.e^{+} e^{-}\right)=5.36 \pm 0.29 \mathrm{keV}{ }^{[4]}$. Here in above calculations the value of $\alpha_{s}\left(m_{c}\right)$ in the QCD radiative correction factor in (14) is chosen to be $0.29^{[3]}$, which is also consistent with our determination from the ratio of $B R(J / \psi \rightarrow 3 g)$ to $B R\left(J / \psi \rightarrow e^{+} e^{-}\right.$) (see below).

Using the experimental data ${ }^{[4]}$

$$
R_{g} \equiv \frac{\Gamma(J / \psi \rightarrow 3 g)}{\Gamma\left(J / \psi \rightarrow e^{+} e^{-}\right)} \approx 10,
$$


and the calculated widths from (14) and (31), (32), we find

$$
\alpha_{s}\left(m_{c}\right)=0.29 \pm 0.02
$$

as campared with the value without relativistic corrections (but with QCD radiative corrections)

$$
\alpha_{s}^{0}\left(m_{c}\right)=0.19 \pm 0.02 .
$$

Clearly, it is the strong suppression due to the relativistic correction to $\mathrm{J} / \psi \rightarrow 3 g$ that enhances the value of $\alpha_{s}\left(m_{c}\right)$ and then makes it consistent with the determined QCD scale parameter $\Lambda_{M S}^{(4)} \approx 200 M e V$ ( for a review see ref.[4]). ( Note that a slightly larger value for $\alpha_{s}\left(m_{c}\right)$ could be obtained if using (27) rather than (31). )

In comparision we have chosen two other groups of parameters and solved the $\mathrm{BS}$ equation for $c \bar{c}$ states by requiring again both their $2 S-1 S$ spacing and $J / \psi-$ $\eta_{c}$ splitting fitting the data. By the same procedure we get two values of $\alpha_{s}\left(m_{c}\right)$ corresponding to the obtained two new wavefunctions of $J / \psi$. With $m_{c}=1.4 \mathrm{GeV}, \lambda=$ $0.24 \mathrm{GeV}^{2}$ and other parameters unchanged (the heavy quarkonia mass spectra are not sensitive to $a$ and $\alpha$ for $\alpha \leq 0.06 G e V)$ we get $\alpha_{s}\left(m_{c}\right)=0.29$. With $m_{c}=1.6 G e V, \lambda=$ $0.22 \mathrm{GeV}^{2}$ and other parameters unchanged we get $\alpha_{s}\left(m_{c}\right)=0.28$.

Meanwhile, we have also solved the nonrelativistic Schrödinger equation for the scalar function $f$ in the nonrelativistic limit by using the same potentials and parametres as (38) and (44), and performing the same calculations, we obtain

$$
\alpha_{s}\left(m_{c}\right)=0.27
$$

Note that in this limit the spin symmetry between the $1^{-}$and $0^{-}$mesons is restored and the relativistic correction from the dynamical sourse is eliminated.

In order to see further the sensitivity of the value of $\alpha_{s}\left(m_{c}\right)$ to the wavefunctions, we have also tried the Gaussian function

$$
f(q)=N \exp \left(-\frac{4 \vec{q}^{2}}{3 q_{0}^{2}}\right),
$$

where $N$ is the normalzation factor, and $q_{0}^{2}$ is the mean value of the momentum squared of the quark inside the meson, which may be roughly estimated by using the scaling law $q_{0}^{2}=m C / 2(C=0.73 G e V)$ found for heavy quarkonia (see, e.g., ref.[10]). Then with $m_{c}=1.5 \mathrm{GeV}$ we find that while the gluonic and leptonic decay widths both become smaller, their ratio does not change very much and gives $\alpha_{s}\left(m_{c}\right)=0.26$.

These results indicate that the determination of $\alpha_{s}\left(m_{c}\right)$ is not sensitive to the dynamical corrections to the wavefunctions, and even not very sensitive to the form of wavefunctions, and the most important contribution comes from the kinematic corrections.

For the $b \bar{b}$ system, with a similar calculation for the decay rates of $\Upsilon \rightarrow 3 g$ and $\Upsilon \rightarrow e^{+} e^{-}$with both relativistic and QCD radiative corrections taken into account, 
and using the observed value of the ratio ${ }^{[4]}$

$$
R_{g} \equiv \frac{\Gamma(\Upsilon \rightarrow 3 g)}{\Gamma\left(\Upsilon \rightarrow e^{+} e^{-}\right)} \approx 32
$$

we find

$$
\alpha_{s}\left(m_{b}\right)=0.20 \pm 0.02,
$$

as compared with the value without relativistic corrections (but with QCD radiative corrections)

$$
\alpha_{s}^{0}\left(m_{b}\right)=0.17 \pm 0.02 .
$$

The value given in (51) agrees with the expected value from the QCD scale parameter. Moreover, with both relativistic and QCD radiative corrections, and $\alpha_{s}\left(m_{b}\right)=0.20$, we get $^{[7]}$

$$
\Gamma\left(\Upsilon \rightarrow e^{+} e^{-}\right)=(1.3 \pm 0.2) K e V,
$$

which is also in agreement with data ${ }^{[4]}$.

We may then conclude that by estimating the relativistic corrections to the gluonic and leptonic decays of heavy quarkonia, we find that the relativistic effects substantially suppress the $V \rightarrow 3 g$ decays, and consequently the determined values of the QCD running coupling constant at the heavy quark mass scale can get enhanced, and can be consistent with other theoretical and experimental expectations.

\section{SUMMARY AND DISCUSSION}

In this paper we calculated the first order relativistic corrections to $V \rightarrow e^{+} e^{-}$and $V \rightarrow 3 g$ for the vector heavy quarkonia $V=J / \psi$ and $\Upsilon$, based on the BS formalism for the decay amplitudes and bound state wavefunctions. We derived the coefficients of the $v^{2} / c^{2}$ term in the decay rates with two different treatments for the on-shell condition of bound quarks, and obtained very similar values for the coefficients. These results may largely reduce the uncertaity in our calculations concerning the on-shell condition in these decay processes. To maintain gauge invariance in the decay amplitudes, the on-shell quarks are certainly better than off-shell quarks. But within the quark-antiquark sector of the Fock states for a heavy quarkonium, gauge invariance may not be guaranteed and other higher Fock states may be needed. Nevertheless, the closeness of the two large negatrive values of the coefficients of the $\vec{q}^{2} / \mathrm{m}^{2}$ term (see (26) and (31)) in the $V \rightarrow 3 g$ widths within the two treatments may encourage us to conclude that the relativistic effects suppress $V \rightarrow 3 g$ decays much more severely than $V \rightarrow e^{+} e^{-}$decays, therefore can make the coupling constants $\alpha_{s}\left(m_{c}\right)$ and $\alpha_{s}\left(m_{b}\right)$ substantially enhanced, as compared with the values obtained without relativistic corrections.

For a more accurate estimate of relativistic corrections, higher orders, e.g., the $\left(v^{2} / c^{2}\right)^{2}$ terms should be taken into account, but this is difficult in our approach, since to the higher orders many other effects e.g. the quark pairs and the dynamical 
gluons may be involved. In connection with higher order effects, we have regularized the singularity associated with the derivative of the wavefunctions at origin in a simple manner, which is valid to the first order of $v^{2} / c^{2}$ but is uncertain to higher order corrections.

We have solved the BS equation for the bound state wavefunctions with QCD inspired inter-quark potentials (linear confinement potential plus one gluon exchange potential) as the BS kernel. With some popular parameters for the potentials we obtained the wavefunctions and used them to calculate the gluonic and leptonic decay widths and their ratios. By comparing the BS wavefunctions with Schrodinger wavefunctions and Gaussian-type wavefunctions, we found that the ratios are not sensitive to the dynamical relativistic effects on the wavefunctions, and even not very sensitive to the form of wavefunctions. We may then conclude that the ratios are insensitive to the dynamical models, and the relativistic effects on the ratios are mainly originated from the kinematic parts of decay amplitudes.

As for the numerical result, using the experimental values of ratio $R_{g} \equiv \frac{\Gamma(V \longrightarrow 3 g)}{\Gamma\left(V \longrightarrow e^{+} e^{-}\right)} \approx$ 10, 32 for $V=J / \psi, \Upsilon$ respectively, and the calculated widths, we found $\alpha_{s}\left(m_{c}\right)=$ $0.29 \pm 0.02$ and $\alpha_{s}\left(m_{b}\right)=0.20 \pm 0.02$. These values for the QCD running coupling constant are consistent with the QCD scale parameter $\Lambda \frac{(4)}{M S} \approx 200 \mathrm{MeV}$.

Recently, there have been some significant progresses in the study of heavy quarkonium decays based on a more fundamental approach of NRQCD (nonrelativistic QCD) (see refs.[11,12]). Many important issues were clarified by this study. It will be interesting to compare their results with ours in connection with the gluonic and leptonic decay widths and the determination of the strong coupling constant at the heavy quark mass scales from these decays.

One of us (K.T.C.) would like to thank the hospitality of G. M. Prosperi and N. Brambilla, the organizers of the International Conference on Quark Confinement and the Hadron Spectrum, Como, Italy, 20-24 June 1994, where this work was briefly reported. He also thanks E. Braaten for sending him the Northwestern University preprints (ref.[11]). This work was supported in part by the National Natural Science Foundation of China, and the State Education Commission of China. 


\section{References}

[1] R. Barbieri, R. Gatto and R. Kögerler, Phys. Lett. B60, 183(1976);

R. Barbieri, R. Gatto and E. Remiddi, Phys. Lett. B61, 465(1976);

R. Barbieri et al., Nucl. Phys. B154, 535(1979); Nucl. Phys. B192, 61(1981).

[2] P. B. Mackenzie and G. P. Lepage, Phys. Rev. lett. 47, 1244(1981).

[3] W. Kwong, P. B. Mackenzie, R. Rosenfeld, and J. L. Rosner, Phys. Rev. D37, 3210(1988).

[4] Particle Data Group, L. Montanet et al., Phys. Rev. D50 (3-I), 1171(1994).

[5] W. Y. Keung and I. J. Muzinich, Phys. Rev. D27, 1518(1983).

[6] M. Consoli and J. H. Field, Phys. Rev. D49, 1293(1994).

[7] K. T. Chao, H. W. Huang, J. H. Liu, and J. Tang, PUTP-94-12;

see also J. Tang, J. H. Liu, and K. T. Chao, Phys. Rev. D (to appear) and references therein.

[8] E. E. Salpeter and H. A. Bethe, Phys. Rev. 84, 1232(1951);

E. E. Salpeter, Phys. Rev. 87, 328(1952).

[9] E. Laermann et al, Phys. Lett. B173, 437(1986);

K. D. Born, Phys. Rev. D 40, 1653 (1989).

[10] C. Quigg and J. L. Rosner, Phys. Rep. 56, 167(1979);

See also W. Kwong, J. L. Rosner, and C. Quigg, Annu. Rev. Nucl. Part. Sci. 37, 325(1987) and references therein for a review of heavy quarkonium phenomenology.

[11] G. T. Bodwin, E. Braaten, and G. P. Lepage, Northwestern University preprint NUHEP-TH-94-5, ANL-HEP-PR-94-24, FERMILAB-PUB-94-073-T;

E. Braaten, Northwestern University preprint NUHEP-TH-94-22.

[12] G. T. Bodwin, E. Braaten, and G. P. Lepage, Phys. Rev. D46, R1914(1992);

W. E. Caswell and G. P. Lepage, Phys. Lett. 167B, 437(1986). 